\title{
In-situ early stage electromigration study in Al line using synchrotron polychromatic X-ray microdiffraction
}

Kai Chen ${ }^{1,2}$, N. Tamura ${ }^{1}$, and K.N. Tu ${ }^{2}$

1 Advanced Light Source, Lawrence Berkeley National Laboratory, 1 Cyclotron Rd, Berkeley CA 94720

2 Department of Materials Science and Engineering, UCLA, Los Angeles, CA 90095 


\begin{abstract}
Electromigration is a phenomenon that has attracted much attention in the semiconductor industry because of its deleterious effects on electronic devices (such as interconnects) as they become smaller and current density passing through them increases. However, the effect of the electric current on the microstructure of interconnect lines during the very early stage of electromigration is not well documented. In the present report, we used synchrotron radiation based polychromatic X-ray microdiffraction for the in-situ study of the electromigration induced plasticity effects on individual grains of an $\mathrm{Al}(\mathrm{Cu})$ interconnect test structure. Dislocation slips which are activated by the electric current stressing are analyzed by the shape change of the diffraction peaks. The study shows polygonization of the grains due to the rearrangement of geometrically necessary dislocations (GND) in the direction of the current. Consequences of these findings are discussed.
\end{abstract}

\title{
INTRODUCTION
}

Electromigration (EM) refers to the mass transport phenomenon of materials due to electric current when the current density reaches high values. This phenomenon can have deleterious effects on microelectronic devices such as integrated circuits (ICs) [1]. In very-largescale integration (VLSI) of circuits on a Si device, $\mathrm{Al}$ or $\mathrm{Cu}$ thin-film line can experience current density as high as $10^{6} \mathrm{~A} / \mathrm{cm}^{2}$, which is enough to cause the atomic motion from the cathode end to the anode end at the device working temperature. Thus voids may form at the cathode end and extrusion may be squeezed out at the anode end, respectively [2]. Although a lot of models have been proposed to understand the mechanism of EM [3-6], the effect of the atomic transport on the dislocation movement and local material microstructure, especially in the early stage of EM is still unclear.

High brilliance X-ray beam with submicron to micron beam size can be produced by focusing optics such as Kirkpatrick-Baez (KB) mirrors at synchrotron facilities [7, 8]. X-ray microdiffraction has been developed for the crystal microstructure study. In this technique, since the crystal grains are larger than the X-ray beam size, a single crystal Laue diffraction pattern is obtained when polychromatic (white beam) X-ray impinges on the sample. By analyzing the Laue diffraction pattern, the orientation and strain information of the material irradiated can be obtained, and the spatial resolution is only limited by the size of the focused X-ray beam.

Previous works have shown the potential of Laue X-ray microdiffraction for EM study on interconnect lines [9-15]. In the present report, we report a more in-depth microstructure study in an $\mathrm{Al}(0.5 \mathrm{wt} \% \mathrm{Cu})$ interconnect during the early stage of electromigration.

\section{EXPERIMENTAL}

The EM test sample is a sputtered $30 \mu \mathrm{m}$ long, $4.1 \mu \mathrm{m}$ wide and $0.75 \mu \mathrm{m}$ thick $\mathrm{Al}(0.5 \mathrm{wt}$

$\% \mathrm{Cu}$ ) two-level structure. A $450 \AA$ thick Ti layer at the bottom of the Al line, as well as another 
layer with $100 \AA$ thick on the top, is used as shunt. The Al line is constrained by a $0.7 \mu \mathrm{m}$ thickness $\mathrm{SiO}_{2}$ passivation layer. Several $\mathrm{W}$ vias are used for electrical connection between this $\mathrm{Al}$ line and unpassivated $\mathrm{Al}(\mathrm{Cu})$ pads at each end. The sample is annealed at $390{ }^{\circ} \mathrm{C}$ in a rough vacuum for $30 \mathrm{~min}$ to stabilize the crystal structure before EM test.

The in-situ X-ray microdiffraction experiment was conducted on beamline 7.3.3 at the Advanced Light Source synchrotron in Berkeley, CA [16]. The X-ray beam size is 0.7 x 0.7 $\mu \mathrm{m}^{2}$. The wired-bonded sample and an X-ray CCD detector are mounted at $45^{\circ}$ and $90^{\circ}$ with respect to the beam, respectively. The CCD detector is fixed at $5 \mathrm{~cm}$ above the sample when white beam Laue diffractions are recorded.

The EM test is conducted at a $224{ }^{\circ} \mathrm{C}$ (heater temperature) and the current density is ramped up to $0.98 \times 10^{6} \mathrm{~A} / \mathrm{cm}^{2}(\mathrm{I}=30 \mathrm{~mA})$ and maintained constant for 45 hours. Before and during the EM test, Laue diffraction patterns are recorded by scanning the sample repeatedly by the submicron-sized white beam. The scanning step size is $0.5 \mu \mathrm{m}$, so 15 steps across the width of the line, 65 steps along the length, and a total of 975 CCD frames are needed to cover the whole surface of the sample for each scan. The exposure time is set as 5 seconds so that the intensity of the diffraction peaks is strong but not saturated. The electronic readout time for each frame is about 10 seconds. As a result, for each scan, it takes about 4 to 5 hours. The Laue diffraction data are analyzed using the custom written XMAS software [11, 17].

\section{RESULTS AND DISCUSSIONS}

\section{$\underline{\text { Dislocation active slip systems }}$}

Large grains in the $\mathrm{Al}$ interconnect line, especially those that span across the width of the line ("bamboo" type), are subject to opposite stress moments on opposite sides across the width. When a certain yield stress was reached, these grains would bend and ultimately polygonize [10].

This aspect of plastic deformation results in broadening of the Laue spots along certain directions compatible with the bending direction due to the arrangement of excess geometrically necessary dislocations (GNDs). Different dislocation slips can result in different shapes of the Laue peaks. As a result, analysis of the Laue spot broadening patterns allows for the identification of the active dislocation glide system that produced the bending.

First the orientation of a given grain is known by indexing the Laue pattern. Secondly, all the possible streaking directions of the Laue peaks are simulated, each of which corresponds to a certain dislocation slip system. Since Al has FCC crystal structure, for each grain, there are 12 possible slip types, all of which are $\{111\} /<110>$ oriented, which means 12 possibilities for both edge dislocations and screw dislocations. Then all of these 24 simulated diffraction patterns are compared with the experimental result, and the best fitting will tell the real dislocation slip type. In figure 1(a), the white streaking peaks are from one of the typical Laue patterns of a deformed grain after 12 hours EM test, and the yellow patterns are the best matched simulation result. In this example, the slip plane is (111); the Burger's vector is along the [101] direction; and the dislocations are edge typed, so the dislocation line direction is perpendicular to the slip plane normal and Burger's vector, which is along [12i] for this case. Figure 1(b) is the pole figure of this grain, with all $<112\rangle$ directions marked and the activated direction circled. In this 
figure, the $\mathrm{x}$ direction is along the electric current flow direction and the $\mathrm{y}$ direction is along the width direction of the $\mathrm{Al}$ line. It is found that the dislocation direction [12i] is the direction belonging to the $\langle 112\rangle$ family and closest to the current flow direction.
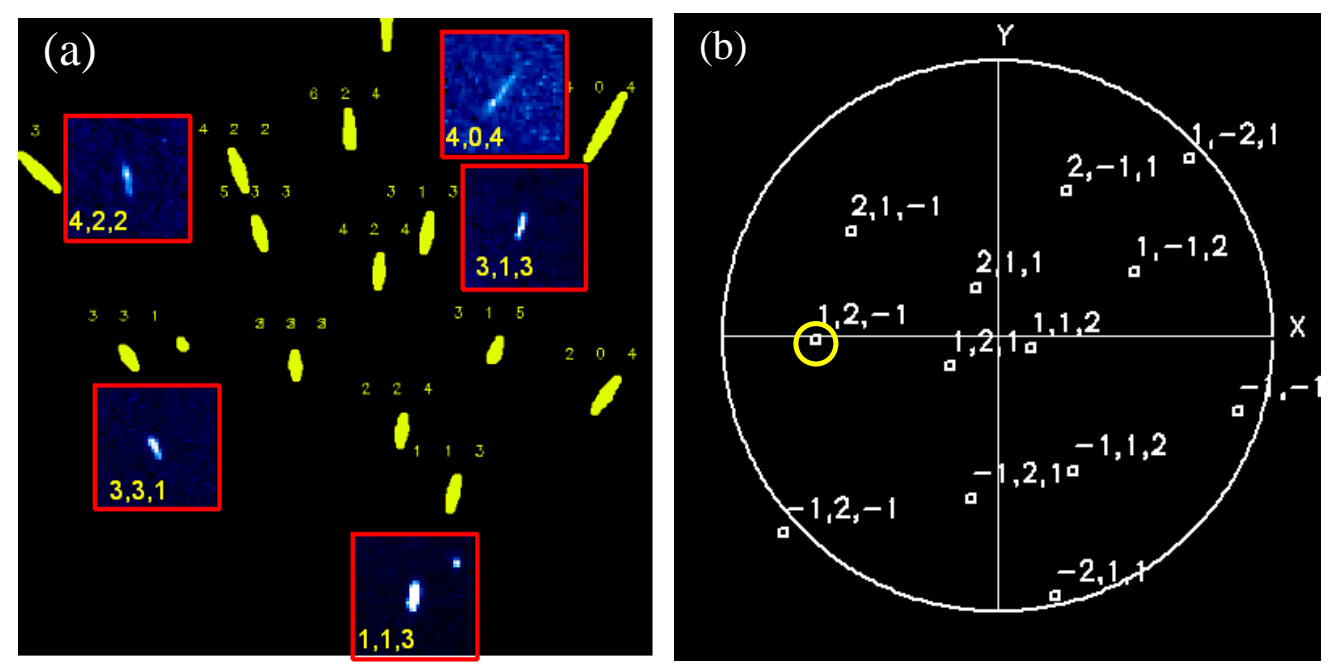

Figure 1. (a) Simulated Laue patterns comparing with the experimental results, and (b) pole figure of this grain with all $<112>$ directions marked

The activated slip systems in 30 grains have been analyzed in this way. All the dislocations are found to be edge typed and about $90 \%$ of the dislocation lines are contained within $5^{\circ}$ with respect to the current flow direction. The dislocations which don't obey this rule are located close to the cathode end. We propose that the alignment of the edge type dislocations along the electric current flow direction is resulted from the minimization of the electrical resistance because the scattering between electrons and dislocations is anisotropic. The dislocations at the cathode end are not aligned regularly because electrons enter the Al line here and the electron motion directions are more disordered. The realignment of dislocations will also influence the atomic diffusion inside the line by affecting the diffusion route. The detailed kinetic study is still underway and will be the subject of a forecoming paper.

At higher tensile stress region, in other words, closer to the cathode, a secondary slip system can be activated, and the Laue patterns are broadened in two different directions, as shown in figure 2(a). This grain is located at about $6 \mu \mathrm{m}$ away from the cathode end. In this example, the two dislocation line directions are [112] and [112] , respectively. The pole figure of this grain, shown as figure 2(b), shows that, as expected, [112] is the direction that is the closest to the current flow direction in $\langle 112\rangle$ family, while [112] is located on the edge of the circle in the pole figure, which represent the XY plane, rather than the second closest direction. At even higher stress level, multiple slip systems are activated. Figure 2(c) is the enlarged (113) peak of a grain locating at about $2 \mu \mathrm{m}$ to the cathode end. The Laue diffraction patterns become complicated since dislocations slip in different orientations, as indexed in the figure. Furthermore, the diffraction peak splitting suggests the formation of subgrains. Since all those subgrains have slightly different orientations, each subpeak in the Laue pattern represents a subgrain in the irradiated volume in the sample. From figure 2(d), which shows all the <112> 
directions in this grain, it is clearly observed that one of the several activated dislocation line directions is along the current direction, while the others are all within the XY plane.
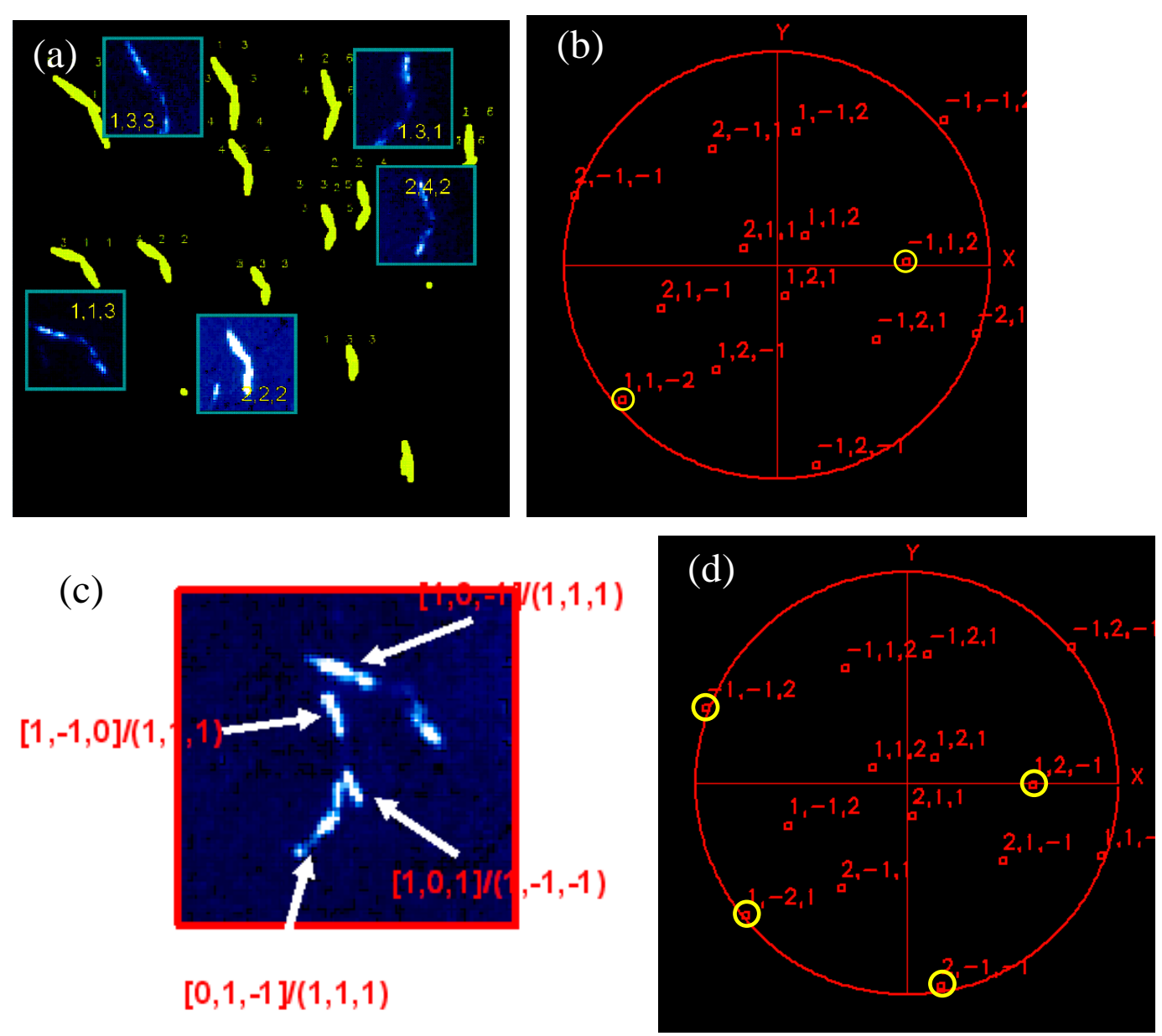

Figure 2. (a) Simulated Laue patterns of a grain which has two slip systems activated and (b) the pole figure of this grain, (c) enlarged (113) diffraction peaks from a grain $2 \mu \mathrm{m}$ to the cathode end showing the multiple activated slip system and subgrain formation and (b) the pole figure of this grain

The secondary slip system, which is within the XY plane, can be roughly explained by the activation of the slip systems of the subgrains formed by EM. Since each of the subgrains has slightly different orientation and is subjected at different local shear stress status, the slip system activated within each subgrain could be different. In other words, the secondary system is activated at a different location in the grain comparing with the primary system. However, the detailed mechanism is not well understood yet.

\section{$\underline{\text { Subgrain formation }}$}

As mentioned above, polygonization happens on large grains close to the cathode by subgrain formations, as indicated by the observation of the subpeaks in some Laue diffraction patterns. In order to study this process, we have tracked the shape evolution of the Laue peaks of 
ten grains in which subgrains were formed. Figure 3 shows a series of (022) diffraction peaks produced by the same grain after different period of EM test. Before the EM test the peak is initially sharp, indicating that there are not many dislocations in the grain. As the EM test continues for 15 hours, the peak is broadened more and more in two directions, which indicates that the grain is bended gradually by the electric current in two different directions, but generally there are no obvious subpeaks in this period. In figure 3(d), the diffraction peak becomes broadened after 21.5 hours current stressing, and three subpeaks are observed, though not very clearly separated. Thus at this stage, the dislocations are partially aligned to form the subgrain boundary. After 25 hours EM test, two subpeaks can be very clearly observed and separated. The separation of the two peaks is larger than the maximum streaking in figure $3(\mathrm{~d})$. Both of the two subpeaks are much sharper than the streaking in figure 3(d), indicating that most of the dislocations are concentrated and aligned at the subgrain boundary.

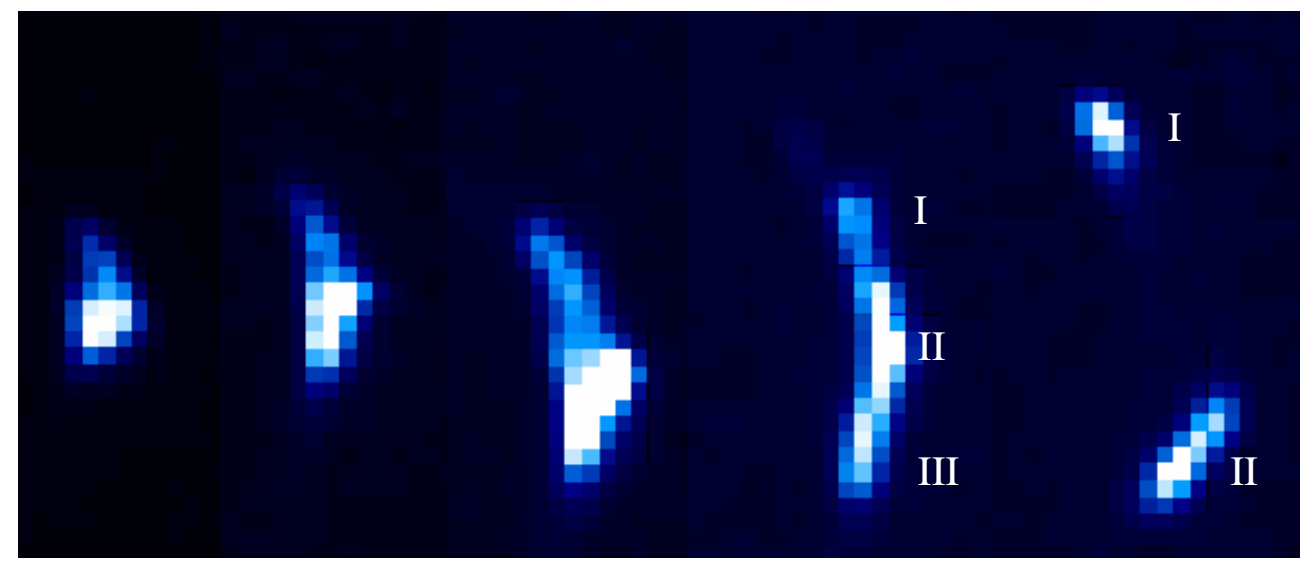

Figure 3. (022) diffraction peaks from the same grain after (a) $0 \mathrm{hr}$, (b) $6.5 \mathrm{hr}$, (c) $15 \mathrm{hr}$, (d) 21.5 $\mathrm{hr}$, and (e) $25 \mathrm{hr}$ EM test

Generally, subgrain forms by the realignment of the dislocations when they reach certain density. More detailed quantitative study, both experimentally and theoretically, is required to understand the kinetics of this procedure.

\section{CONCLUSION}

In this paper, using polychromatic X-ray beam microdiffraction, we have studied the plastic deformation of the Al interconnect line induced by EM. The activated slip systems have been studied from the shape change of the diffraction peaks. It is found that all the dislocations are edge typed. With relative low stress, only the slip system with the dislocation line direction which is the closest to electric current flow direction is activated while under high stress above certain critical value, more slip system can be activated besides the one along the current direction, and the secondary ones prefer to be within the XY plane. Furthermore, the evolution of the diffraction peaks has been carefully studied. The bending of the crystal plane indicated by the elongation of the diffraction peaks and resulted from the rearrangement of the geometrically necessary dislocations results in the peak splitting and subgrain boundary formation by the realignment of the dislocations. 


\section{ACKNOWLEDGEMENTS}

The Advanced light Source is supported by the Director, Office of Science, Office of Basic Energy Sciences, of the U.S. Department of Energy under Contract No. DE-AC0205CH11231 at Lawrence Berkeley National Laboratory

\section{REFERENCES}

1. I. A. Blech, J. Appl. Phys. 47, 1203 (1976)

2. K. N. Tu, J. Appl. Phys. 94, 5451 (2003)

3. S. Vaidya, and A. K. Sinda, Thin Solid Films 75, 253 (1981)

4. P. S. Ho, and T. Kwok, Rep. Prog. Phys. 52, 301 (1989)

5. M. A Korhonen, P. Borgesen, K. N. Tu, and C. Li, J. Appl. Phys. 73, 3790 (1993)

6. H. Gan, K. N. Tu, J. Appl. Phys. 97, 063517 (2005)

7. O. Hignette, P. Cloetens, G. Rostaing, P. Bernard, and C. Morawe, Review of Scientific Instruments 76 : Art. No. 063709 (2005)

8. W. J. Liu, G. E. Ice, J. Z. Tischler, A. Khounsary, C. Liu, L. Assoufid, and A. T. Macrander, Rev. Sci. Instrum. 76: Art. No. 113701 (2005)

9. P. C. Wang, G. S. Cargill III, I. C. Noynan, and C. K. Hu, Appl. Phys. Lett. 72, 1296 (1998)

10. B. C. Valek, J. C. Bravman, N. Tamura, A. A. MacDowell, R. S. Celestre, H. A. Padmore, R. Spolenak, W. L. Brown, B. W. Batterman, and J. R. Patel, Appl. Phys. Lett. 81, 4168 (2002)

11. N. Tamura, H. A Padmore, and J. R. Patel, Mat. Sci. Eng. A, 399, 92 (2005)

12. B. C. Valek, N. Tamura, R. Spolenak, W. A Caldwell, A. A. MacDowell, R. S. Celestre, H. A. Padmore, J. C. Bravman, B. W. Batterman, W. D. Nix, and J. R. Patel, J. Appl. Phys. 94, 3757 (2003)

13. A. S. Budiman, N. Tamura, B. C. Valek, K. Gadre, J. Maiz, R. Spolenak, J. R. Patel, and W. D. Nix, Mater. Res. Soc. Symp. Proc. 914 (2006)

14. R. I. Barabash, G. E. Ice, N. Tamura, B. C. Valek, J. C. Bravman, R. Spolenak, and J. R. Patel, J. Appl. Phys. 93, 5701 (2003)

15. A. S. Budiman, W. D. Nix, N. Tamura, B. C. Valek, K. Gadre, J. Maiz, R. Spolenak, J. R. Patel, Appl. Phys. Lett. 88, 233515 (2006)

16. N. Tamura, A. A. MacDowell, R. Spolenak, B. C. Valek, J. C. Bravman, W. L. Brown, R. S. Celestre, H. A. Padmore, B. W. Batterman and J. R. Patel, J. of Synchrotron Radiat. 10 (2003) 137-143.

17. N. Tamura, R. Spolenak, B. C. Valek, A. Manceau, M. Meier Chang, R. S. Celestre, A. A. MacDowell, H. A. Padmore, and J. R. Patel, Rev. Sci. Instrum. 73, 1369 (2002) 